\title{
Qualitative behavior of a class of second order nonlinear differential equations on the halfline
}

\author{
by Svatoslav StanĚK (Olomouc)
}

\begin{abstract}
A differential equation of the form $\left(q(t) k(u) u^{\prime}\right)^{\prime}=F(t, u) u^{\prime}$ is considered and solutions $u$ with $u(0)=0$ are studied on the halfline $[0, \infty)$. Theorems about the existence, uniqueness, boundedness and dependence of solutions on a parameter are given.
\end{abstract}

1. Introduction. In [6] the differential equation

$$
\left(k(u) u^{\prime}\right)^{\prime}=f(t) u^{\prime},
$$

where $k, f \in C^{0}\left(\mathbb{R}_{+}\right), \mathbb{R}_{+}=[0, \infty)$, was considered and the author gave sufficient conditions for the existence and uniqueness of nonnegative solutions $u$ such that $u(0)=0, u(t)>0$ for $t>0$. This problem is connected with the description of the mathematical model of the infiltration of water. For more details see e.g. [3]-[5]. Special cases of (1) were considered in [1], [2], [4] and [5]. In [7] the differential equation

$$
\left(q(t) k(u) u^{\prime}\right)^{\prime}=f(t) h(u) u^{\prime},
$$

where $q, k, f, h \in C^{0}\left(\mathbb{R}_{+}\right)$, was considered and sufficient conditions for the existence, uniqueness and boundedness of nonnegative solutions of (2) on $\mathbb{R}_{+}$ were given. Simultaneously the dependence of solutions of the differential equation

$$
\left(q(t) k(u) u^{\prime}\right)^{\prime}=\lambda f(t) h(u) u^{\prime}, \quad \lambda>0,
$$

on the positive parameter $\lambda$ was studied.

In this paper we consider the differential equation

$$
\left(q(t) k(u) u^{\prime}\right)^{\prime}=F(t, u) u^{\prime}
$$

1991 Mathematics Subject Classification: 34A34, 34C11, 34B15, 45G10.

Key words and phrases: nonlinear differential equation, nonnegative solution, nonpositive solution, existence and uniqueness of solutions, bounded solution, dependence of solutions on a parameter, boundary value problem. 
where $q \in C^{0}\left(\mathbb{R}_{+}\right), k \in C^{0}(\mathbb{R}), F \in C^{0}\left(\mathbb{R}_{+} \times \mathbb{R}\right)$, which is a generalization of (2), and study solutions $u$ of this equation on $\mathbb{R}_{+}$such that $u(0)=0$. We give assumptions under which any nontrivial solution of (4) is either strictly increasing or strictly decreasing on $\mathbb{R}_{+}$and discuss the existence, uniqueness and boundedness of solutions. In the last section we consider the dependence of solutions of the differential equation

$$
\left(q(t) k(u) u^{\prime}\right)^{\prime}=\lambda F(t, u) u^{\prime}, \quad \lambda>0,
$$

on the positive parameter $\lambda$ and study the boundary value problem $\left(4_{\lambda}\right)$, $\lim _{t \rightarrow \infty} u(t, \lambda)=a(\in \mathbb{R}-\{0\})$. Our theorems imply all results of [6] and [7]. The proofs of existence theorems are based on the Tikhonov-Schauder fixed point theorem, on the iterative method and on the monotone behavior of some operators. The proofs of uniqueness are different from the ones in [6] and [7].

2. Notations, lemmas. Consider the differential equation (4), where $q, k, F$ satisfy the following assumptions $\left(\mathbb{R}_{+}=[0, \infty), \mathbb{R}_{-}=(-\infty, 0]\right)$ :

$\left(\mathrm{H}_{1}\right) \quad q \in C^{0}\left(\mathbb{R}_{+}\right), q(t)>0$ for $t \in(0, \infty), \quad \int_{0} \frac{d s}{q(s)}<\infty ;$

$\left(\mathrm{H}_{2}\right) \quad k \in C^{0}(\mathbb{R}), k(u) u>0$ for $u \in \mathbb{R}, u \neq 0 ;$

$\left(\mathrm{H}_{3}\right) \quad \int_{0} \frac{k(s)}{s} d s<\infty, \quad \int^{0} \frac{k(s)}{s} d s<\infty$,

$$
\int_{-\infty} \frac{k(s)}{s} d s=\infty, \quad \int^{\infty} \frac{k(s)}{s} d s=\infty ;
$$

$\left(\mathrm{H}_{4}\right) \quad F \in C^{0}\left(\mathbb{R}_{+} \times \mathbb{R}\right), f_{1}(t) h_{1}(u) \leq F(t, u) \leq f_{2}(t) h_{2}(u)$

for $(t, u) \in \mathbb{R}_{+} \times \mathbb{R}$, where

$f_{i} \in C^{0}\left(\mathbb{R}_{+}\right), 0<f_{i}(t)$ for $t \in \mathbb{R}_{+}, f_{i}$ is decreasing on $\mathbb{R}_{+}$,

$h_{i} \in C^{0}(\mathbb{R}), h_{i}(u) u \geq 0$ for $u \in \mathbb{R}$ and

$H_{i}(u):=\int_{0}^{u} h_{i}(x) d x$ is strictly increasing on $\mathbb{R}_{+}$,

$T_{i}(u):=\int_{u}^{0} h_{i}(x) d x$ is strictly increasing on $\mathbb{R}_{-}(i=1,2) ;$

$\left(\mathrm{H}_{5}\right) \quad \int_{0} \frac{k(x)}{H_{i}(x)} d x<\infty, \quad \int^{0} \frac{k(x)}{T_{i}(x)} d x<\infty$,

$\int^{\infty} \frac{k(x)}{H_{i}(x)} d x=\infty, \quad \int_{-\infty} \frac{k(x)}{T_{i}(x)} d x=\infty \quad(i=1,2)$. 
By a solution of (4) we mean a function $u \in C^{0}\left(\mathbb{R}_{+}\right)$which is continuously differentiable on $(0, \infty)$ and such that $u(0)=0, \lim _{t \rightarrow 0_{+}} q(t) k(u(t)) u^{\prime}(t)$ $=0, q(t) k(u(t)) u^{\prime}(t)$ is continuously differentiable on $(0, \infty)$ and $(4)$ is satisfied on $(0, \infty)$.

Remark 1. It follows from $\left(\mathrm{H}_{2}\right)$ and $\left(\mathrm{H}_{4}\right)$ that $k(0)=0, h_{i}(0)=0$ $(i=1,2)$, and $F(t, 0)=0$ for $t \in \mathbb{R}_{+}$. Therefore $u=0$ is a solution of (4).

LEMma 1. Let $u(t)$ be a nontrivial solution of (4). Then either $u^{\prime}(t)>0$ on $(0, \infty)$ or $u^{\prime}(t)<0$ on $(0, \infty)$.

Proof. Let $u$ be a solution of (4), $u \neq 0$. Then

$$
q(t) k(u(t)) u^{\prime}(t)=\int_{0}^{t} F(s, u(s)) u^{\prime}(s) d s \quad \text { for } t \in(0, \infty) .
$$

Assume there exist $0<t_{1}<t_{2}$ such that $u^{\prime}\left(t_{1}\right)=u^{\prime}\left(t_{2}\right)=0, u^{\prime}(t) \neq 0$ for $t \in\left(t_{1}, t_{2}\right)$. Suppose $u^{\prime}(t)>0$ on $\left(t_{1}, t_{2}\right)$. If $u(t)>0$ (resp. $\left.<0\right)$ on $\left(t_{1}, t_{2}\right)$, then

$$
\begin{aligned}
\int_{t_{1}}^{t_{2}} f_{1}(s) h_{1}(u(s)) u^{\prime}(s) d s & \leq \int_{t_{1}}^{t_{2}} F(s, u(s)) u^{\prime}(s) d s \quad(=0) \\
& \leq \int_{t_{1}}^{t_{2}} f_{2}(s) h_{2}(u(s)) u^{\prime}(s) d s
\end{aligned}
$$

which contradicts

$$
\begin{gathered}
\int_{t_{1}}^{t_{2}} f_{1}(s) h_{1}(u(s)) u^{\prime}(s) d s \geq f_{1}\left(t_{2}\right) \int_{u\left(t_{1}\right)}^{u\left(t_{2}\right)} h_{1}(s) d s>0 \\
\text { resp. } \left.\int_{t_{1}}^{t_{2}} f_{2}(s) h_{2}(u(s)) u^{\prime}(s) d s \leq f_{2}\left(t_{2}\right) \int_{u\left(t_{1}\right)}^{u\left(t_{2}\right)} h_{2}(s) d s<0\right) .
\end{gathered}
$$

Therefore $u(\xi)=0$ for a $\xi \in\left(t_{1}, t_{2}\right)$ and then $u(t)<0$ on $\left[t_{1}, \xi\right)$ and

$$
\int_{t_{1}}^{\xi} F(s, u(s)) u^{\prime}(s) d s \leq \int_{t_{1}}^{\xi} f_{2}(s) h_{2}(u(s)) u^{\prime}(s) d s \leq f_{2}(\xi) \int_{u\left(t_{1}\right)}^{0} h_{2}(s) d s<0
$$

which contradicts $0=\int_{t_{1}}^{\xi} F(s, u(s)) u^{\prime}(s) d s$. We can easily check that $u^{\prime}(t)<$ 0 on $\left(t_{1}, t_{2}\right)$ is also impossible.

Assume $u^{\prime}(\tau)=0$ for a $\tau \in(0, \infty)$ and $u^{\prime}(t) \neq 0$ for $t \in(0, \tau)$. If $u^{\prime}(t)>0$ (resp. $\left.u^{\prime}(t)<0\right)$ on $(0, \tau)$, then $u(t)>0$ (resp. $\left.u(t)<0\right)$ on this interval and

$$
\int_{0}^{\tau} F(s, u(s)) u^{\prime}(s) d s \geq \int_{0}^{\tau} f_{1}(s) h_{1}(u(s)) u^{\prime}(s) d s \geq f_{1}(\tau) \int_{0}^{u(\tau)} h_{1}(s) d s>0
$$




$$
\begin{aligned}
\text { (resp. } \int_{0}^{\tau} F(s, u(s)) u^{\prime}(s) d s & \geq \int_{0}^{\tau} f_{2}(s) h_{2}(u(s)) u^{\prime}(s) d s \\
& \left.\geq f_{2}(\tau) \int_{0}^{u(\tau)} h_{2}(s) d s>0\right),
\end{aligned}
$$

which contradicts $0=\int_{0}^{\tau} F(s, u(s)) u^{\prime}(s) d s$.

This proves $u^{\prime}(t) \neq 0$ on $(0, \infty)$ and since $u \in C^{1}((0, \infty))$ we have either $u^{\prime}(t)>0$ on $(0, \infty)$ or $u^{\prime}(t)<0$ on $(0, \infty)$.

R e m a r k 2. It follows from Lemma 1 that $u \in \mathcal{A}_{+} \cup \mathcal{A}_{-}$for any nontrivial solution $u$ of (4), where

$$
\begin{aligned}
& \mathcal{A}_{+}=\left\{u \in C^{0}\left(\mathbb{R}_{+}\right): u(0)=0, u \text { is strictly increasing on } \mathbb{R}_{+}\right\}, \\
& \mathcal{A}_{-}=\left\{u \in C^{0}\left(\mathbb{R}_{+}\right): u(0)=0, u \text { is strictly decreasing on } \mathbb{R}_{+}\right\} .
\end{aligned}
$$

Set

$$
W_{+}(u)=\int_{0}^{u} k(s) d s \quad \text { for } u \in \mathbb{R}_{+}, \quad W_{-}(u)=\int_{0}^{u} k(s) d s \quad \text { for } u \in \mathbb{R}_{-} .
$$

Obviously, $W_{+}$is strictly increasing on $\mathbb{R}_{+}$, and $W_{-}$is strictly decreasing on $\mathbb{R}_{-}$.

LEMma 2. If $u$ is a solution of (4), $u \neq 0$, then $u$ is a solution of the integral equation

$$
u(t)=W_{+}^{-1}\left(\int_{0}^{t} \frac{1}{q(s)} \int_{0}^{u(s)} F\left(u^{-1}(x), x\right) d x d s\right)
$$

or the integral equation

$$
u(t)=W_{-}^{-1}\left(-\int_{0}^{t} \frac{1}{q(s)} \int_{u(s)}^{0} F\left(u^{-1}(x), x\right) d x d s\right)
$$

in the set $\mathcal{A}_{+}$or $\mathcal{A}_{-}$, respectively. Conversely, if $u \in \mathcal{A}_{\varepsilon}, \varepsilon \in\{+,-\}$, is a solution of $\left(6_{\varepsilon}\right)$, then $u$ is a solution of $(4)$ and $u \neq 0$. Here $W_{\varepsilon}^{-1}$ and $u^{-1}$ denote the inverse functions to $W_{\varepsilon}$ and $u$, respectively.

Proof. Let $u \neq 0$ be a solution of (4). Then $u \in \mathcal{A}_{+} \cup \mathcal{A}_{-}$by Remark 2 and (5) holds. If $u \in \mathcal{A}_{+}$(resp. $\left.u \in \mathcal{A}_{-}\right)$, then

$$
\begin{aligned}
W_{+}(u(t)) & =\int_{0}^{t} \frac{1}{q(s)} \int_{0}^{s} F(x, u(x)) u^{\prime}(x) d x d s \\
& =\int_{0}^{t} \frac{1}{q(s)} \int_{0}^{u(s)} F\left(u^{-1}(x), x\right) d x d s
\end{aligned}
$$




$$
\begin{aligned}
\left(\operatorname{resp} . W_{-}(u(t))\right. & =\int_{0}^{t} \frac{1}{q(s)} \int_{0}^{s} F(x, u(x)) u^{\prime}(x) d x d s \\
& \left.=-\int_{0}^{t} \frac{1}{q(s)} \int_{u(s)}^{0} F\left(u^{-1}(x), x\right) d x d s\right)
\end{aligned}
$$

for $t \in \mathbb{R}_{+}$and $u$ is a solution of $\left(6_{+}\right)$or $\left(6_{-}\right)$in $\mathcal{A}_{+}$or $\mathcal{A}_{-}$, respectively.

Conversely, let $u \in \mathcal{A}_{\varepsilon}, \varepsilon \in\{+,-\}$, be a solution of $\left(6_{\varepsilon}\right)$. Then $u(0)=0$, $u \in C^{0}\left(\mathbb{R}_{+}\right), u$ is continuously differentiable on $(0, \infty)$,

$$
\lim _{t \rightarrow 0_{+}} q(t) k(u(t)) u^{\prime}(t)=\lim _{t \rightarrow 0_{+}} \int_{0}^{u(t)} F\left(u^{-1}(s), s\right) d s=0
$$

and $\left(q(t) k(u(t)) u^{\prime}(t)\right)^{\prime}=F(t, u(t)) u^{\prime}(t)$ for $t>0$. Consequently, $u$ is a solution of (4) and $u \neq 0$.

Remark 3. It follows from Lemma 2 that solving (4) is equivalent to solving $\left(6_{+}\right)$and $\left(6_{-}\right)$in $\mathcal{A}_{+}$and $\mathcal{A}_{-}$, respectively.

Set

$$
\begin{aligned}
K_{j}(u)=\int_{0}^{u} \frac{k(s)}{H_{j}(s)} d s \quad \text { for } u \in \mathbb{R}_{+}, \\
V_{j}(u)=\int_{u}^{0} \frac{k(s)}{T_{j}(s)} d s \quad \text { for } u \in \mathbb{R}_{-}, j=1,2,
\end{aligned}
$$

and

$$
\begin{aligned}
k_{1}(t) & =\int_{0}^{t} \frac{f_{1}(s)}{q(s)} d s, & k_{2}(t) & =f_{2}(0) \int_{0}^{t} \frac{d s}{q(s)}, \\
l_{1}(t) & =f_{1}(0) \int_{0}^{t} \frac{d s}{q(s)}, & l_{2}(t) & =\int_{0}^{t} \frac{f_{2}(s)}{q(s)} d s, \\
\underline{\varphi}_{+}(t) & =K_{1}^{-1}\left(k_{1}(t)\right), & \bar{\varphi}_{+}(t) & =K_{2}^{-1}\left(k_{2}(t)\right), \\
\underline{\varphi}_{-}(t) & =V_{1}^{-1}\left(l_{1}(t)\right), & \bar{\varphi}_{-}(t) & =V_{2}^{-1}\left(l_{2}(t)\right) \quad \text { for } t \in \mathbb{R}_{+} .
\end{aligned}
$$

Obviously, $\lim _{u \rightarrow \infty} K_{j}(u)=\infty, \lim _{u \rightarrow-\infty} V_{j}(u)=\infty(j=1,2)$ by $\left(\mathrm{H}_{5}\right)$.

Lemma 3. If $u \in \mathcal{A}_{\varepsilon}$ is a solution of $\left(6_{\varepsilon}\right), \varepsilon \in\{+,-\}$, then

$$
\underline{\varphi_{\varepsilon}}(t) \leq u(t) \leq \bar{\varphi}_{\varepsilon}(t) \quad \text { for } t \in \mathbb{R}_{+}
$$

and 


$$
\begin{aligned}
& \left(9_{+}\right) \quad u\left(t_{2}\right)-u\left(t_{1}\right) \geq f_{1}\left(t_{2}\right) H_{1}\left(\underline{\varphi}_{+}\left(t_{1}\right)\right) \int_{t_{1}}^{t_{2}} \frac{d t}{q(t)} \\
& \times\left[\max \left\{k(x): \underline{\varphi}_{+}\left(t_{1}\right) \leq x \leq \bar{\varphi}_{+}\left(t_{2}\right)\right\}\right]^{-1} \quad \text { for } u \in \mathcal{A}_{+} \text {and } 0<t_{1}<t_{2}, \\
& \left(9_{-}\right) \quad u\left(t_{1}\right)-u\left(t_{2}\right) \geq-f_{2}\left(t_{2}\right)\left(T_{2}\left(\bar{\varphi}_{-}\left(t_{1}\right)\right) \int_{t_{1}}^{t_{2}} \frac{d t}{q(t)}\right.
\end{aligned}
$$

$\times\left[\max \left\{-k(x): \underline{\varphi_{-}}\left(t_{2}\right) \leq x \leq \bar{\varphi}_{-}\left(t_{1}\right)\right\}\right]^{-1} \quad$ for $u \in \mathcal{A}_{-}$and $0<t_{1}<t_{2}$.

Proof. Let $u \in \mathcal{A}_{+}$be a solution of $\left(6_{+}\right)$. Then $\left(\right.$cf. $\left.\left(\mathrm{H}_{4}\right)\right)$

$$
\begin{aligned}
f_{1}(t) \int_{0}^{u(t)} h_{1}(s) d s & \leq q(t) k(u(t)) u^{\prime}(t) \quad\left(=\int_{0}^{u(t)} F\left(u^{-1}(s), s\right) d s\right) \\
& \leq f_{2}(0) \int_{0}^{u(t)} h_{2}(s) d s,
\end{aligned}
$$

hence

$$
\begin{aligned}
& \frac{f_{1}(t)}{q(t)} \leq\left(\int_{0}^{u(t)} \frac{k(s)}{H_{1}(s)} d s\right)^{\prime}, \\
& \frac{f_{2}(0)}{q(t)} \geq\left(\int_{0}^{u(t)} \frac{k(s)}{H_{2}(s)} d s\right)^{\prime} \quad \text { for } t \in(0, \infty),
\end{aligned}
$$

and integrating (10) from 0 to $t$, we obtain

$$
\begin{gathered}
\int_{0}^{t} \frac{f_{1}(s)}{q(s)} d s \leq \int_{0}^{u(t)} \frac{k(s)}{H_{1}(s)} d s=K_{1}(u(t)), \\
f_{2}(0) \int_{0}^{t} \frac{d s}{q(s)} \geq \int_{0}^{u(t)} \frac{k(s)}{H_{2}(s)} d s=K_{2}(u(t)) .
\end{gathered}
$$

Consequently,

$$
\underline{\varphi}_{+}(t) \leq u(t) \leq \bar{\varphi}_{+}(t) \quad \text { for } t \in \mathbb{R}_{+} .
$$

Let $0<t_{1}<t_{2}$. Then

$$
\begin{aligned}
W_{+}\left(u\left(t_{2}\right)\right)-W_{+}\left(u\left(t_{1}\right)\right) & =\int_{t_{1}}^{t_{2}} \frac{1}{q(s)} \int_{0}^{u(s)} F\left(u^{-1}(x), x\right) d x d s \\
& \geq \int_{t_{1}}^{t_{2}} \frac{1}{q(s)} \int_{0}^{u(s)} f_{1}\left(u^{-1}(x)\right) h_{1}(x) d x d s
\end{aligned}
$$




$$
\begin{aligned}
& \geq f_{1}\left(t_{2}\right) \int_{t_{1}}^{t_{2}} \frac{d s}{q(s)} \int_{0}^{\underline{\varphi_{+}}} h_{1}^{\left(t_{1}\right)} h_{1}(s) d s \\
& =f_{1}\left(t_{2}\right) H_{1}\left(\underline{\varphi}_{+}\left(t_{1}\right)\right) \int_{t_{1}}^{t_{2}} \frac{d s}{q(s)}
\end{aligned}
$$

and since

$$
u\left(t_{2}\right)-u\left(t_{1}\right)=\frac{1}{k(\xi)}\left(W_{+}\left(u\left(t_{2}\right)\right)-W_{+}\left(u\left(t_{1}\right)\right)\right),
$$

where $\xi \in\left(u\left(t_{1}\right), u\left(t_{2}\right)\right) \subset\left(\underline{\varphi}_{+}\left(t_{1}\right), \bar{\varphi}_{+}\left(t_{2}\right)\right)$, we see that $\left(9_{+}\right)$is true.

Let $u \in \mathcal{A}_{-}$be a solution of $\left(6_{-}\right)$. Then $\left(\mathrm{cf} .\left(\mathrm{H}_{4}\right)\right)$

$$
\begin{aligned}
-f_{2}(t) \int_{u(t)}^{0} h_{2}(s) d s & \leq q(t) k(u(t)) u^{\prime}(t) \quad\left(=\int_{0}^{u(t)} F\left(u^{-1}(s), s\right) d s\right) \\
& \leq-f_{1}(0) \int_{u(t)}^{0} h_{1}(s) d s
\end{aligned}
$$

hence

$$
\begin{aligned}
& \frac{f_{2}(t)}{q(t)} \leq\left(\int_{u(t)}^{0} \frac{k(s)}{T_{2}(s)} d s\right)^{\prime}, \\
& \frac{f_{1}(0)}{q(t)} \geq\left(\int_{u(t)}^{0} \frac{k(s)}{T_{1}(s)} d s\right)^{\prime} \quad \text { for } t \in(0, \infty),
\end{aligned}
$$

and integrating the last inequalities from 0 to $t$, we obtain

$$
\begin{gathered}
\int_{0}^{t} \frac{f_{2}(s)}{q(s)} d s \leq \int_{u(t)}^{0} \frac{k(s)}{T_{2}(s)} d s=V_{2}(u(t)), \\
f_{1}(0) \int_{0}^{t} \frac{d s}{q(s)} \geq \int_{u(t)}^{0} \frac{k(s)}{T_{1}(s)} d s=V_{1}(u(t)) .
\end{gathered}
$$

Consequently,

$$
\underline{\varphi}_{-}(t) \leq u(t) \leq \bar{\varphi}_{-}(t) \quad \text { for } t \in \mathbb{R}_{+} .
$$

Let $0<t_{1}<t_{2}$. Then

$$
\begin{aligned}
W_{-}\left(u\left(t_{2}\right)\right)-W_{-}\left(u\left(t_{1}\right)\right) & =-\int_{t_{1}}^{t_{2}} \frac{1}{q(s)} \int_{u(s)}^{0} F\left(u^{-1}(x), x\right) d x d s \\
& \geq-\int_{t_{1}}^{t_{2}} \frac{1}{q(s)} \int_{u(s)}^{0} f_{2}\left(u^{-1}(x)\right) h_{2}(x) d x d s
\end{aligned}
$$




$$
\begin{aligned}
& \geq-f_{2}\left(t_{2}\right) \int_{t_{1}}^{t_{2}} \frac{d s}{q(s)} \int_{\bar{\varphi}_{-}\left(t_{1}\right)}^{0} h_{2}(s) d s \\
& =-f_{2}\left(t_{2}\right) T_{2}\left(\bar{\varphi}_{-}\left(t_{1}\right)\right) \int_{t_{1}}^{t_{2}} \frac{d s}{q(s)},
\end{aligned}
$$

and since

$$
u\left(t_{1}\right)-u\left(t_{2}\right)=-(1 / k(\xi))\left(W_{-}\left(u\left(t_{2}\right)\right)-W_{-}\left(u\left(t_{1}\right)\right)\right),
$$

where $\xi \in\left(u\left(t_{2}\right), u\left(t_{1}\right)\right) \subset\left(\underline{\varphi}_{-}\left(t_{2}\right), \bar{\varphi}_{-}\left(t_{1}\right)\right)$, we see that $\left(9_{-}\right)$is true.

Set $\mathcal{K}_{\varepsilon}=\left\{u \in \mathcal{A}_{\varepsilon}: \underline{\varphi}_{\varepsilon}(t) \leq u(t) \leq \bar{\varphi}_{\varepsilon}(t)\right.$ for $t \in \mathbb{R}_{+}, u$ satisfies $\left.\left(9_{\varepsilon}\right)\right\}$, and define $\mathrm{T}_{\varepsilon}: \mathcal{K}_{\varepsilon} \rightarrow C^{0}\left(\mathbb{R}_{+}\right)$by

$$
\left(\mathrm{T}_{\varepsilon} u\right)(t)=W_{\varepsilon}^{-1}\left(\int_{0}^{t} \frac{1}{q(s)} \int_{0}^{u(s)} F\left(u^{-1}(x), x\right) d x d s\right) \quad \varepsilon \in\{+,-\} .
$$

Lemma 4. $\mathrm{T}_{\varepsilon}: \mathcal{K}_{\varepsilon} \rightarrow \mathcal{K}_{\varepsilon}$ for each $\varepsilon \in\{+,-\}$.

Proof. We will prove $\mathrm{T}_{+}: \mathcal{K}_{+} \rightarrow \mathcal{K}_{+}$(the proof of $\mathrm{T}_{-}: \mathcal{K}_{-} \rightarrow \mathcal{K}_{-}$is very similar and will be omitted). Let $u \in \mathcal{K}_{+}$. Setting

$$
\begin{aligned}
& \alpha(t)=\int_{0}^{t} \frac{1}{q(s)} \int_{0}^{u(s)} F\left(u^{-1}(x), x\right) d x d s-W_{+}\left(\underline{\varphi}_{+}(t)\right), \\
& \beta(t)=\int_{0}^{t} \frac{1}{q(s)} \int_{0}^{u(s)} F\left(u^{-1}(x), x\right) d x d s-W_{+}\left(\bar{\varphi}_{+}(t)\right)
\end{aligned}
$$

for $t \in \mathbb{R}_{+}$, we have

$$
\begin{aligned}
\alpha^{\prime}(t) & =\frac{1}{q(t)} \int_{0}^{u(t)} F\left(u^{-1}(x), x\right) d x-k\left(\underline{\varphi}_{+}(t)\right) \underline{\varphi}_{+}^{\prime}(t) \\
& =\frac{1}{q(t)}\left[\int_{0}^{u(t)} F\left(u^{-1}(x), x\right) d x-f_{1}(t) H_{1}\left(\underline{\varphi}_{+}(t)\right)\right] \\
& \geq \frac{1}{q(t)}\left[\int_{0}^{u(t)} f_{1}\left(u^{-1}(x)\right) h_{1}(x) d x-f_{1}(t) \int_{0}^{\underline{\varphi}_{+}} h_{1}(x) d x\right] \\
& \geq \frac{1}{q(t)} \int_{0}^{u(t)}\left(f_{1}\left(u^{-1}(x)\right)-f_{1}(t)\right) h_{1}(x) d x \geq 0,
\end{aligned}
$$




$$
\begin{aligned}
\beta^{\prime}(t) & =\frac{1}{q(t)} \int_{0}^{u(t)} F\left(u^{-1}(x), x\right) d x-k\left(\bar{\varphi}_{+}(t)\right) \bar{\varphi}_{+}^{\prime}(t) \\
& =\frac{1}{q(t)}\left[\int_{0}^{u(t)} F\left(u^{-1}(x), x\right) d x-f_{2}(0) H_{2}\left(\bar{\varphi}_{+}(t)\right)\right] \\
& \leq \frac{1}{q(t)}\left[\int_{0}^{u(t)} f_{2}\left(u^{-1}(x)\right) h_{2}(x) d x-f_{2}(0) \int_{0}^{\bar{\varphi}_{+}(t)} h_{2}(x) d x\right] \\
& \leq \frac{1}{q(t)} \int_{0}^{u(t)}\left(f_{2}\left(u^{-1}(x)\right)-f_{2}(0)\right) h_{2}(x) d x \leq 0
\end{aligned}
$$

for $t \in(0, \infty)$. Since $\alpha(0)=\beta(0)=0$ and $\alpha^{\prime}(t) \geq 0, \beta^{\prime}(t) \leq 0$ on $(0, \infty)$, we have $\alpha(t) \geq 0, \beta(t) \leq 0$ and consequently,

$$
\underline{\varphi}_{+}(t) \leq\left(\mathrm{T}_{+} u\right)(t) \leq \bar{\varphi}_{+}(t) \quad \text { for } t \in \mathbb{R}_{+} \text {and } u \in \mathcal{K}_{+} .
$$

Let $0<t_{1}<t_{2}$ and let $u \in \mathcal{K}_{+}$. Then

$$
\begin{aligned}
W_{+}\left(\left(\mathrm{T}_{+} u\right)\left(t_{2}\right)\right)-W_{+}\left(\left(\mathrm{T}_{+} u\right)\left(t_{1}\right)\right) & =\int_{t_{1}}^{t_{2}} \frac{1}{q(s)} \int_{0}^{u(s)} F\left(u^{-1}(x), x\right) d x d s \\
& \geq \int_{t_{1}}^{t_{2}} \frac{1}{q(s)} \int_{0}^{u(s)} f_{1}\left(u^{-1}(x)\right) h_{1}(x) d x d s \\
& \geq \int_{t_{1}}^{t_{2}} \frac{f_{1}(s)}{q(s)} H_{1}(u(s)) d s \\
& \geq f_{1}\left(t_{2}\right) H_{1}\left(\underline{\varphi}_{+}\left(t_{1}\right)\right) \int_{t_{1}}^{t_{2}} \frac{d s}{q(s)}
\end{aligned}
$$

and

$$
\begin{aligned}
& W_{+}\left(\left(\mathrm{T}_{+} u\right)\left(t_{2}\right)\right)-W_{+}\left(\left(\mathrm{T}_{+} u\right)\left(t_{1}\right)\right)=k(\xi)\left[\left(\mathrm{T}_{+} u\right)\left(t_{2}\right)-\left(\mathrm{T}_{+} u\right)\left(t_{1}\right)\right] \\
& \leq\left[\left(\mathrm{T}_{+}\right)\left(t_{2}\right)-\left(\mathrm{T}_{+} u\right)\left(t_{1}\right)\right] \max \left\{k(u): \underline{\varphi}_{+}\left(t_{1}\right) \leq u \leq \bar{\varphi}_{+}\left(t_{2}\right)\right\}
\end{aligned}
$$

(here $\left.\xi \in\left(\left(\mathrm{T}_{+} u\right)\left(t_{1}\right),\left(\mathrm{T}_{+} u\right)\left(t_{2}\right)\right) \subset\left(\underline{\varphi}_{+}\left(t_{1}\right), \bar{\varphi}_{+}\left(t_{2}\right)\right)\right)$, hence

$$
\begin{aligned}
& \left(\mathrm{T}_{+} u\right)\left(t_{2}\right)-\left(\mathrm{T}_{+} u\right)\left(t_{1}\right) \\
& \geq f_{1}\left(t_{2}\right) H_{1}\left(\underline{\varphi}_{+}\left(t_{1}\right)\right) \int_{t_{1}}^{t_{2}} \frac{d s}{q(s)}\left[\max \left\{k(u): \underline{\varphi}_{+}\left(t_{1}\right) \leq u \leq \bar{\varphi}_{+}\left(t_{2}\right)\right\}\right]^{-1} .
\end{aligned}
$$

From (11) and (12) it follows that $\mathrm{T}_{+} u \in \mathcal{K}_{+}$, therefore $\mathrm{T}_{+}: \mathcal{K}_{+} \rightarrow \mathcal{K}_{+}$. 


\section{Existence theorems}

THEOREM 1. Let assumptions $\left(\mathrm{H}_{1}\right)-\left(\mathrm{H}_{5}\right)$ be satisfied. Then a solution $u \in \mathcal{A}_{\varepsilon}$ of (4) exists for each $\varepsilon \in\{+,-\}$.

Pro of. By Lemma 2, $u \in \mathcal{A}_{\varepsilon}$ is a solution of (4) if and only if $u$ is a fixed point of the operator $\mathrm{T}_{\varepsilon}$. We will prove that under assumptions $\left(\mathrm{H}_{1}\right)-\left(\mathrm{H}_{5}\right)$ a fixed point of $T_{+}$exists. The existence of a fixed point of $T_{-}$can be proved similarly.

Let $\mathbf{X}$ be the Fréchet space of $C^{0}$-functions on $\mathbb{R}_{+}$with the topology of uniform convergence on compact subintervals of $\mathbb{R}_{+}$. Then $\mathcal{K}_{+}$is a bounded closed convex subset of $\mathbf{X}$ and $\mathrm{T}_{+}: \mathcal{K}_{+} \rightarrow \mathcal{K}_{+}$(see Lemma 4 ) is a continuous operator. It follows from the inequalities $\left(0 \leq t_{1}<t_{2}, u \in \mathcal{K}_{+}\right)$

$$
\begin{aligned}
0 & \leq W_{+}\left(\left(\mathrm{T}_{+} u\right)\left(t_{2}\right)\right)-W_{+}\left(\left(\mathrm{T}_{+} u\right)\left(t_{1}\right)\right)=\int_{t_{1}}^{t_{2}} \frac{1}{q(s)} \int_{0}^{u(s)} F\left(u^{-1}(x), x\right) d x d s \\
& \leq \int_{t_{1}}^{t_{2}} \frac{1}{q(s)} \int_{0}^{u(s)} f_{2}\left(u^{-1}(x)\right) h_{2}(x) d x d s \leq f_{2}(0) H_{2}\left(\bar{\varphi}_{+}\left(t_{2}\right)\right) \int_{t_{1}}^{t_{2}} \frac{d s}{q(s)}
\end{aligned}
$$

and from the Arzelà-Ascoli theorem that $\mathrm{T}_{+}\left(\mathcal{K}_{+}\right)$is a relatively compact subset of $\mathbf{X}$. According to the Tikhonov-Schauder fixed point theorem there exists a fixed point $u_{+}$of $\mathrm{T}_{+}$.

THEOREM 2. Let assumptions $\left(\mathrm{H}_{1}\right)-\left(\mathrm{H}_{5}\right)$ be satisfied and suppose that $\left(\mathrm{H}_{6}\right) \quad F(\cdot, u) \operatorname{sign}(u)$ is decreasing on $\mathbb{R}_{+}$for each fixed $u \in \mathbb{R}$.

Then there exist solutions $u_{\varepsilon}, v_{\varepsilon} \in \mathcal{A}_{\varepsilon}$ of (4) for each $\varepsilon \in\{+,-\}$ such that $u_{\varepsilon}(t) \leq v_{\varepsilon}(t)$ for $t \in \mathbb{R}_{+}$. Moreover,

$$
u_{\varepsilon}(t) \leq u(t) \leq v_{\varepsilon}(t), \quad t \in \mathbb{R}_{+}, \varepsilon \in\{+,-\},
$$

for any solution $u \in \mathcal{A}_{\varepsilon}$ of (4) and

$$
u(t) \neq v(t), \quad t>0,
$$

for any two different solutions $u, v$ of (4).

Proof. Let assumptions $\left(\mathrm{H}_{1}\right)-\left(\mathrm{H}_{6}\right)$ be satisfied and let $u \in \mathcal{A}_{+}$be a solution of (4). Define sequences $\left\{u_{n}\right\} \subset \mathcal{A}_{+},\left\{v_{n}\right\} \subset \mathcal{A}_{+}$by the recurrence formulas

$$
\begin{array}{ll}
u^{[0]}=\underline{\varphi}_{+}, & u^{[n+1]}=\mathrm{T}_{+}\left(u^{[n]}\right), \\
v^{[0]}=\bar{\varphi}_{+}, \quad v^{[n+1]}=\mathrm{T}_{+}\left(v^{[n]}\right),
\end{array}
$$

for $n \in \mathbb{N}$. Then $u^{[0]}(t) \leq u(t) \leq v^{[0]}(t)$ on $\mathbb{R}_{+}$by Lemma 3 and $u^{[0]}(t) \leq$ $u^{[1]}(t) \leq v^{[0]}(t), u^{[0]}(t) \leq v^{[1]}(t) \leq v^{[0]}(t)$ on $\mathbb{R}_{+}$by Lemma 4. Since $\alpha, \beta \in$ 
$\mathcal{A}_{+}, \alpha(t) \leq \beta(t)$ for $t \in \mathbb{R}_{+}$implies

$$
\begin{aligned}
\left(\mathrm{T}_{+} \alpha\right)(t) & =W_{+}^{-1}\left(\int_{0}^{t} \frac{1}{q(s)} \int_{0}^{\alpha(t)} F\left(\alpha^{-1}(x), x\right) d x d s\right) \\
& \leq W_{+}^{-1}\left(\int_{0}^{t} \frac{1}{q(s)} \int_{0}^{\beta(t)} F\left(\beta^{-1}(x), x\right) d x d s\right)=\left(\mathrm{T}_{+} \beta\right)(t)
\end{aligned}
$$

on $\mathbb{R}_{+}$and $\mathrm{T}_{+}: \mathcal{K}_{+} \rightarrow \mathcal{K}_{+}$by Lemma 4 , we deduce

$$
\begin{aligned}
u^{[0]}(t) & \leq u^{[1]}(t) \leq \ldots \leq u^{[n]}(t) \\
& \leq \ldots \leq u(t) \leq \ldots \leq v^{[n]}(t) \leq \ldots \leq v^{[1]}(t) \leq v^{[0]}(t)
\end{aligned}
$$

for $t \in \mathbb{R}_{+}$and $n \in \mathbb{N}$. Therefore the limits $\lim _{n \rightarrow \infty} u^{[n]}(t)=: u_{+}(t)$, $\lim _{n \rightarrow \infty} v^{[n]}(t)=: v_{+}(t)$ exist for all $t \geq 0, u_{+}(t) \leq u(t) \leq v_{+}(t)$ on $\mathbb{R}_{+}$and using the Lebesgue dominated convergence theorem we see that $u_{+}, v_{+} \in \mathcal{K}_{+}$ are solutions of $\left(6_{+}\right)$and thus also solutions of (4) by Lemma 2.

Let $u, v \in \mathcal{A}_{+}$be different solutions of (4). First, suppose there exists a $t_{1}>0$ such that $u(t)<v(t)$ for $t \in\left(0, t_{1}\right)$ and $u\left(t_{1}\right)=v\left(t_{1}\right)$. Then

$$
\begin{aligned}
0 & =W_{+}\left(v\left(t_{1}\right)\right)-W_{+}\left(u\left(t_{1}\right)\right) \\
& =\int_{0}^{t_{1}} \frac{1}{q(s)}\left[\int_{0}^{v(s)} F\left(v^{-1}(x), x\right) d x-\int_{0}^{u(s)} F\left(u^{-1}(x), x\right) d x\right] d s \\
& \geq \int_{0}^{t_{1}} \frac{1}{q(s)}\left[\int_{0}^{u(s)}\left(F\left(v^{-1}(x), x\right)-F\left(u^{-1}(x), x\right)\right) d x\right] d s
\end{aligned}
$$

and since (cf. $\left.\left(\mathrm{H}_{6}\right)\right) F\left(v^{-1}(x), x\right)-F\left(u^{-1}(x), x\right) \geq 0$ for $x \in\left[0, u\left(t_{1}\right)\right]$ we have necessarily $F\left(v^{-1}(x), x\right)-F\left(u^{-1}(x), x\right)=0$ for $x \in\left[0, u\left(t_{1}\right)\right]$. Then

$$
W_{+}\left(v\left(t_{1}\right)\right)-W_{+}\left(u\left(t_{1}\right)\right)=\int_{0}^{t_{1}} \frac{1}{q(s)} \int_{u(s)}^{v(s)} F\left(v^{-1}(x), x\right) d x d s>0,
$$

which contradicts $W_{+}\left(v\left(t_{1}\right)\right)-W_{+}\left(u\left(t_{1}\right)\right)=0$.

Secondly, assume there exist $0<t_{1}<t_{2}$ such that $u\left(t_{1}\right)=v\left(t_{1}\right), u\left(t_{2}\right)=$ $v\left(t_{2}\right), u(t) \neq v(t)$ for $t \in\left(t_{1}, t_{2}\right)$, for example assume

$$
u(t)>v(t) \quad \text { for } t \in\left(t_{1}, t_{2}\right) .
$$

Then $u^{\prime}\left(t_{1}\right) \geq v^{\prime}\left(t_{1}\right), u^{\prime}\left(t_{2}\right) \leq v^{\prime}\left(t_{2}\right)$ and

$$
\begin{aligned}
0 & \leq q\left(t_{1}\right) k\left(u\left(t_{1}\right)\right)\left(u^{\prime}\left(t_{1}\right)-v^{\prime}\left(t_{1}\right)\right)-q\left(t_{2}\right) k\left(u\left(t_{2}\right)\right)\left(u^{\prime}\left(t_{2}\right)-v^{\prime}\left(t_{2}\right)\right) \\
& =\int_{u\left(t_{2}\right)}^{u\left(t_{1}\right)}\left[F\left(u^{-1}(x), x\right)-F\left(v^{-1}(x), x\right)\right] d x .
\end{aligned}
$$


On the other hand, since $u\left(t_{2}\right)>u\left(t_{1}\right)$ and $\left(\mathrm{cf} .\left(\mathrm{H}_{6}\right)\right) F\left(u^{-1}(x), x\right)-$ $F\left(v^{-1}(x), x\right) \geq 0$ on $\left[u\left(t_{1}\right), u\left(t_{2}\right)\right]$ we have

$$
\int_{u\left(t_{2}\right)}^{u\left(t_{1}\right)}\left[F\left(u^{-1}(x), x\right)-F\left(v^{-1}(x), x\right)\right] d x \leq 0,
$$

therefore in virtue of (15), $u^{\prime}\left(t_{1}\right)=v^{\prime}\left(t_{1}\right), u^{\prime}\left(t_{2}\right)=v^{\prime}\left(t_{2}\right)$ and $F\left(u^{-1}(x), x\right)-$ $F\left(v^{-1}(x), x\right)=0$ for $x \in\left[u\left(t_{1}\right), u\left(t_{2}\right)\right]$. Then

$$
\begin{aligned}
0 & =\left(W_{+}\left(u\left(t_{2}\right)\right)-W_{+}\left(u\left(t_{1}\right)\right)\right)-\left(W_{+}\left(v\left(t_{2}\right)\right)-W_{+}\left(v\left(t_{1}\right)\right)\right) \\
& =\int_{t_{1}}^{t_{2}} \frac{1}{q(s)} \int_{v(s)}^{u(s)} F\left(u^{-1}(x), x\right) d x d s
\end{aligned}
$$

and since $H_{1}(u(t))-H_{1}(v(t))>0$ on $\left(t_{1}, t_{2}\right)$,

$$
\begin{aligned}
\int_{t_{1}}^{t_{2}} \frac{1}{q(s)} \int_{v(s)}^{u(s)} F\left(u^{-1}(x), x\right) d x d s & \geq f_{1}\left(t_{2}\right) \int_{t_{1}}^{t_{2}} \frac{1}{q(s)} \int_{v(s)}^{u(s)} h_{1}(x) d x d s \\
& =f_{1}\left(t_{2}\right) \int_{t_{1}}^{t_{2}} \frac{1}{q(s)}\left(H_{1}(u(s))-H_{1}(v(s))\right) d s>0
\end{aligned}
$$

which contradicts $(16)$. Hence $u(t) \neq v(t)$ for $t \in(0, \infty)$. For $\varepsilon=-$ the proof is similar.

\section{Bounded solutions}

THEOREM 3. Let assumptions $\left(\mathrm{H}_{1}\right)-\left(\mathrm{H}_{5}\right)$ be satisfied. Then

(i) any nontrivial solution of $(4)$ is bounded (on $\mathbb{R}_{+}$) if and only if $\int_{0}^{\infty} d s / q(s)<\infty$

(ii) any nontrivial solution of (4) is unbounded (on $\mathbb{R}_{+}$) if and only if $\int_{0}^{\infty} d s / q(s)=\infty$.

Pro of. We will prove our theorem for $\varepsilon=+$ (the case $\varepsilon=-$ is similar). First observe that either $\int_{0}^{\infty} d s / q(s)<\infty$ or $\int_{0}^{\infty} d s / q(s)=\infty$.

(a) If $\int_{0}^{\infty} d s / q(s)<\infty$ then by Lemma 3 any solution of (4) is bounded.

(b) Let $\int_{0}^{\infty} d s / q(s)=\infty$ and $u \in \mathcal{A}_{+}$be a solution of (4). Then $u \neq 0$, $W_{+}(u(t))$

$$
=\int_{0}^{t} \frac{1}{q(s)} \int_{0}^{u(s)} F\left(u^{-1}(x), x\right) d x d s \geq \int_{0}^{t} \frac{1}{q(s)} \int_{0}^{u(s)} f_{1}\left(u^{-1}(x)\right) h_{1}(x) d x d s
$$

and since $0 \not \equiv\left(\int_{0}^{u(s)} f_{1}\left(u^{-1}(x)\right) h_{1}(x) d x\right)^{\prime} \geq 0$ on $\mathbb{R}_{+}$we see $\lim _{t \rightarrow \infty} W_{+}(u(t))$ $=\infty$, hence $\lim _{t \rightarrow \infty} u(t)=\infty$. 
Let $u \in \mathcal{A}_{+}$be a solution of (4). Since $u$ is strictly increasing on $\mathbb{R}_{+}$(by Lemma 1 ), $u$ is either bounded or unbounded on $\mathbb{R}_{+}$. Accordingly, either $\int_{0}^{\infty} d s / q(s)<\infty$ or $\int_{0}^{\infty} d s / q(s)=\infty$ by (b) and (a) above.

5. Uniqueness theorems. Let assumptions $\left(\mathrm{H}_{1}\right)-\left(\mathrm{H}_{5}\right)$ be satisfied. By Theorem 1 equation (4) has at least one solution in $\mathcal{A}_{+}$and at least one solution in $\mathcal{A}_{-}$. Since $u=0$ is also a solution of (4), we see that under assumptions $\left(\mathrm{H}_{1}\right)-\left(\mathrm{H}_{5}\right)$ equation (4) admits at least three different solutions. In the next theorems we will give sufficient conditions for the existence of just three different solutions of (4). that

ThEOREM 4. Let assumptions $\left(\mathrm{H}_{1}\right)-\left(\mathrm{H}_{5}\right)$ be satisfied. Moreover, assume

$\left(\mathrm{H}_{7}\right) \quad$ There exists an $\varepsilon>0$ such that:

(i) $\left|F\left(t_{1}, u\right)-F\left(t_{2}, u\right)\right| \leq L\left|t_{1}-t_{2}\right| \min \left\{\left|h_{1}(u)\right|,\left|h_{2}(u)\right|\right\}$ for $\left(t_{i}, u\right)$ $\in[0, \varepsilon] \times[-\varepsilon, \varepsilon](i=1,2)$, where $L>0$ is a constant,

(ii) the modulus of continuity $\gamma(t)\left(:=\sup \left\{\left|q\left(t_{1}\right)-q\left(t_{2}\right)\right|: t_{1}, t_{2} \in\right.\right.$ $\left.\left.[0, \varepsilon],\left|t_{1}-t_{2}\right| \leq t\right\}\right)$ of $q$ on $[0, \varepsilon]$ satisfies

$$
\limsup _{t \rightarrow 0_{+}} \gamma(t) / t<\infty
$$

$\left(\mathrm{H}_{8}\right) \quad$ The function $F(t, u)$ locally satisfies a Lipschitz condition on $(0, \infty)$ $\times(\mathbb{R}-\{0\})$ with respect to $t$.

Then equation (4) admits just three different solutions.

Proof. Assume $u_{1}, u_{2} \in \mathcal{A}_{+}$are solutions of (4) and assume on the contrary $u_{1} \neq u_{2}$. First, we will prove $u_{1}(t)=u_{2}(t)$ on an interval $[0, b]$, $b>0$. Setting $A_{j}=\lim _{t \rightarrow \infty} u_{j}(t)$ and $w_{j}=u_{j}^{-1}(j=1,2)$, we see $0<A_{j} \leq$ $\infty, w_{j}:\left[0, A_{j}\right) \rightarrow \mathbb{R}_{+}$are continuous strictly increasing functions and

$$
w_{j}^{\prime}(x)=k(x) q\left(w_{j}(x)\right)\left[\int_{0}^{x} F\left(w_{j}(s), s\right) d s\right]^{-1} \quad \text { for } x \in\left(0, A_{j}\right), j=1,2 .
$$

Hence

$$
w_{j}(x)=\int_{0}^{x} k(s) q\left(w_{j}(s)\right)\left[\int_{0}^{s} F\left(w_{j}(t), t\right) d t\right]^{-1} d s \quad \text { for } x \in\left(0, A_{j}\right), j=1,2,
$$

and thus for $x \in\left[0, \min \left(A_{1}, A_{2}\right)\right)$ we have

$$
\begin{aligned}
& \text { (17) } w_{1}(x)-w_{2}(x)=\int_{0}^{x}\left(q\left(w_{1}(s)\right)-q\left(w_{2}(x)\right)\right) k(s)\left[\int_{0}^{s} F\left(w_{2}(t), t\right) d t\right]^{-1} d s \\
& +\int_{0}^{x} \frac{q\left(w_{1}(s)\right) k(s)}{\int_{0}^{s} F\left(w_{1}(t), t\right) d t \int_{0}^{s} F\left(w_{2}(t), t\right) d t} \int_{0}^{s}\left(F\left(w_{2}(t), t\right)-F\left(w_{1}(t), t\right)\right) d t d s .
\end{aligned}
$$


Let $\varepsilon>0$ be as in assumption $\left(\mathrm{H}_{7}\right)$ and set $a=\min \left\{u_{1}(\varepsilon), u_{2}(\varepsilon)\right\}, X(x)=$ $\max \left\{\left|w_{1}(s)-w_{2}(s)\right|: 0 \leq s \leq x\right\}$ for $x \in[0, a]$. Suppose $X(x)>0$ for $x \in(0, a]$. Then (cf. $\left.\left(\mathrm{H}_{7}\right)\right)$

$$
\left|q\left(w_{1}(x)\right)-q\left(w_{2}(x)\right)\right| \leq \gamma(X(x)) \quad \text { for } x \in[0, a]
$$

and using $\left(\mathrm{H}_{7}\right)$ and (17) we have

$$
\begin{aligned}
& \left|w_{1}(x)-w_{2}(x)\right| \leq \int_{0}^{x} \frac{\gamma(X(s)) k(s)}{\int_{0}^{s} f_{1}\left(w_{2}(t)\right) h_{1}(t) d t} d s \\
& +L \int_{0}^{x} \frac{q\left(w_{1}(s)\right) k(s)}{\int_{0}^{s} f_{1}\left(w_{1}(t)\right) h_{1}(t) d t \int_{0}^{s} f_{1}\left(w_{2}(t)\right) h_{1}(t) d t} \int_{0}^{s}\left|w_{2}(t)-w_{1}(t)\right| h_{1}(t) d t d s \\
& \leq \frac{\gamma(X(x))}{f_{1}(\varepsilon)} K_{1}(x)+L X(x) \frac{K_{1}(x)}{f_{1}^{2}(\varepsilon)} \max \{q(t): 0 \leq t \leq \varepsilon\}
\end{aligned}
$$

for $0 \leq x \leq a$. Hence

$$
X(x) \leq(B \gamma(X(x))+C X(x)) K_{1}(x), \quad x \in[0, a],
$$

where $B=1 / f_{1}(\varepsilon), C=B^{2} L \max \{q(t): 0 \leq t \leq \varepsilon\}$, and therefore

$$
\frac{\gamma(X(x))}{X(x)} K_{1}(x) \geq(1 / B)\left(1-C K_{1}(x)\right), \quad x \in(0, a] .
$$

Then $\left(\right.$ cf. $\left.\left(\mathrm{H}_{7}\right)\right)$

$$
\lim _{x \rightarrow 0_{+}} \frac{\gamma(X(x))}{X(x)} K_{1}(x)=0,
$$

which contradicts $\lim _{x \rightarrow 0_{+}}(1 / B)\left(1-C K_{1}(x)\right)=1 / B>0$. This proves $u_{1}(t)=u_{2}(t)$ for $t \in[0, b]$ with a positive number $b$.

Secondly, assume $[0, c]$ is the maximal interval where $u_{1}(t)=u_{2}(t)$. Define $Y(t)=\max \left\{\left|u_{2}(s)-u_{1}(s)\right|: c \leq s \leq t\right\}, \alpha(t)=\min \left\{u_{1}(t), u_{2}(t)\right\}$, $\beta(t)=\max \left\{u_{1}(t), u_{2}(t)\right\}$ for $t \geq c$. Then $Y(c)=0, \alpha(c)=\beta(c), 0 \leq$ $\beta(t)-\alpha(t) \leq Y(t)>0$ for $t>c$. From the equality $(t \geq c)$

$$
\begin{aligned}
W_{+}\left(u_{2}(t)\right) & -W_{+}\left(u_{1}(t)\right) \\
= & \int_{c}^{t} \frac{1}{q(s)}\left[\int_{u_{1}(c)}^{u_{2}(s)} F\left(u_{2}^{-1}(x), x\right) d x-\int_{u_{2}(c)}^{u_{1}(s)} F\left(u_{1}^{-1}(x), x\right) d x\right] d s
\end{aligned}
$$

it follows that

$$
\begin{aligned}
W_{+}\left(u_{2}(t)\right)-W_{+}\left(u_{1}(t)\right)= & \int_{c}^{t} \frac{1}{q(s)}\left[\int_{u_{1}(c)}^{u_{2}(s)}\left(F\left(u_{2}^{-1}(x), x\right)-F\left(u_{1}^{-1}(x), x\right)\right) d x\right. \\
& \left.+\int_{u_{2}(s)}^{u_{1}(s)} F\left(u_{1}^{-1}(x), x\right) d x\right] d s \quad \text { for } t \in[c, \infty) .
\end{aligned}
$$


By assumption $\left(\mathrm{H}_{8}\right)$ there exist positive numbers $\varepsilon_{0}$ and $K$ such that $\left|F\left(t_{1}, u\right)-F\left(t_{2}, u\right)\right| \leq K\left|t_{1}-t_{2}\right|$ for $\left(t_{j}, u\right) \in\left[c, c+\varepsilon_{0}\right] \times\left[u_{1}(c), u_{1}(c)+\varepsilon_{0}\right]$. Set $\varepsilon=\min \left\{\varepsilon_{0}, \beta^{-1}\left(\varepsilon_{0}+u_{1}(c)\right)-c\right\}, m=\min \left\{u_{1}^{\prime}(x): c \leq x \leq \alpha^{-1}(\beta(c+\varepsilon))\right\}$ (>0 by Lemma 1), $M=\max \left\{F\left(u_{1}^{-1}(x), x\right): \alpha(c) \leq x \leq \beta(c+\varepsilon)\right\}$. Then $\left|F\left(u_{2}^{-1}(x), x\right)-F\left(u_{1}^{-1}(x), x\right)\right| \leq K\left|u_{2}^{-1}(x)-u_{1}^{-1}(x)\right| \quad$ for $x \in[\alpha(c), \alpha(c+\varepsilon)]$ and for $x \in[c, c+\varepsilon]$ we have

$$
\begin{array}{r}
\left|u_{1}^{-1}\left(u_{2}(x)\right)-x\right|=\left|u_{1}^{-1}\left(u_{2}(x)\right)-u_{1}^{-1}\left(u_{1}(x)\right)\right|=\left|\left(u_{1}^{-1}\right)^{\prime}(\xi)\right|\left|u_{2}(x)-u_{1}(x)\right| \\
=\left(1 /\left|u_{1}^{\prime}(\eta)\right|\right)\left|u_{2}(x)-u_{1}(x)\right| \leq(1 / m)\left|u_{2}(x)-u_{1}(x)\right| \leq Y(x) / m,
\end{array}
$$

where $\xi \in(\alpha(x), \beta(x))$ and $\eta=u_{1}^{-1}(\xi) \in\left[u_{1}^{-1}(\alpha(x)), u_{1}^{-1}(\beta(x))\right] \subset$ $\left[c, \alpha^{-1}(\beta(c+\varepsilon))\right]$. Consequently,

$$
\left|u_{1}^{-1}(x)-u_{2}^{-1}(x)\right| \leq(1 / m) Y\left(u_{2}^{-1}(x)\right) \quad \text { for } x \in\left[u_{1}(c), u_{2}(c+\varepsilon)\right] .
$$

Therefore $\left(r=\max \left\{u_{2}^{\prime}(t): c \leq t \leq c+\varepsilon\right\}\right)$

$$
\begin{aligned}
& \left|W_{+}\left(u_{2}(t)\right)-W_{+}\left(u_{1}(t)\right)\right| \\
& \quad \leq \int_{c}^{t} \frac{1}{q(s)}\left[K \int_{u_{1}(c)}^{u_{2}(s)}\left|u_{2}^{-1}(x)-u_{1}^{-1}(x)\right| d x+\int_{\alpha(s)}^{\beta(s)} F\left(u_{1}^{-1}(x), x\right) d x\right] d s \\
& \quad \leq \int_{c}^{t} \frac{1}{q(s)}\left[K \int_{u_{1}(c)}^{u_{2}(s)}\left(Y\left(u_{2}^{-1}(x)\right) / m\right) d x+M(\beta(s)-\alpha(s))\right] d s \\
& \quad \leq \int_{c}^{t} \frac{1}{q(s)}\left[(r K / m) \int_{c}^{s} Y(x) d x+M Y(s)\right] d s \\
& \quad \leq \int_{c}^{t} \frac{1}{q(s)}[(K r(s-c) / m)+M] Y(s) d s \leq((K \varepsilon r / m)+M) Y(t) \int_{c}^{t} \frac{d s}{q(s)}
\end{aligned}
$$

for $t \in[c, c+\varepsilon]$. Since $(t \in[c, c+\varepsilon])$

$$
\left|W_{+}\left(u_{2}(t)\right)-W_{+}\left(u_{1}(t)\right)\right|=k(\xi)\left|u_{2}(t)-u_{1}(t)\right|,
$$

where $\xi \in(\alpha(t), \beta(t)) \subset[\alpha(c), \beta(c+\varepsilon)]$, we have

$$
\left|u_{2}(t)-u_{1}(t)\right| \leq[(K \varepsilon r / m)+M](Y(t) / p) \int_{c}^{t} \frac{d s}{q(s)},
$$

with $p=\min \{k(u): \alpha(c) \leq u \leq \beta(c+\varepsilon)\}(>0)$, hence

$$
Y(t) \leq[(K \varepsilon r / m)+M](Y(t) / p) \int_{c}^{t} \frac{d s}{q(s)}, \quad t \in[c, c+\varepsilon] .
$$


Then

$$
1 \leq[(K \varepsilon r / m)+M](1 / p) \int_{c}^{t} \frac{d s}{q(s)},
$$

for $c<t \leq c+\varepsilon$, which is impossible. This proves $u_{1}(t)=u_{2}(t)$ for $t \in \mathbb{R}_{+}$. The uniqueness of solutions of (4) in $\mathcal{A}_{-}$can be treated quite analogously.

TheOREm 5. Suppose that assumptions $\left(\mathrm{H}_{1}\right)-\left(\mathrm{H}_{7}\right)$ are satisfied. Then equation (4) admits just three different solutions.

Proof. It is sufficient to prove that under assumptions $\left(\mathrm{H}_{1}\right)-\left(\mathrm{H}_{7}\right), u_{\varepsilon}=$ $v_{\varepsilon}, \varepsilon=+,-$, where $u_{\varepsilon}, v_{\varepsilon}$ are defined in Theorem 2. If for example, $u_{+} \neq v_{+}$ then $u_{+}(t)<v_{+}(t)$ on $(0, \infty)$ by Theorem 2 . Since assumptions $\left(\mathrm{H}_{1}\right)-\left(\mathrm{H}_{5}\right)$ and $\left(\mathrm{H}_{7}\right)$ imply (see the first part of the proof of Theorem 4) $u_{+}(t)=v_{+}(t)$ on an interval $[0, b](b>0)$, we have a contradiction.

6. Dependence of solutions on a parameter. Consider the differential equation

$$
\left(q(t) k(u) u^{\prime}\right)^{\prime}=\lambda F(t, u) u^{\prime}, \quad \lambda>0,
$$

depending on the positive parameter $\lambda$.

Theorem 6. Suppose that assumptions $\left(\mathrm{H}_{1}\right)-\left(\mathrm{H}_{6}\right)$ are satisfied. Then for each $\varepsilon \in\{+,-\}$ there exist solutions $u_{\varepsilon}(t ; \lambda), v_{\varepsilon}(t ; \lambda)$ of $\left(18_{\lambda}\right)$ such that $u_{\varepsilon}(t ; \lambda) \leq v_{\varepsilon}(t ; \lambda)$ for $t \in \mathbb{R}_{+}$. Moreover ,

$$
u_{\varepsilon}(t ; \lambda) \leq u(t ; \lambda) \leq v_{\varepsilon}(t ; \lambda), \quad t \in \mathbb{R}_{+},
$$

for any solution $u(t ; \lambda) \in \mathcal{A}_{\varepsilon}$ of $\left(18_{\lambda}\right)$ and

$$
\begin{array}{ll}
u_{+}\left(t ; \lambda_{1}\right)<u_{+}\left(t ; \lambda_{2}\right), & v_{+}\left(t ; \lambda_{1}\right)<v_{+}\left(t ; \lambda_{2}\right), \\
u_{-}\left(t ; \lambda_{1}\right)>u_{-}\left(t ; \lambda_{2}\right), & v_{-}\left(t ; \lambda_{1}\right)>v_{-}\left(t, \lambda_{2}\right)
\end{array}
$$

for all $t \in \mathbb{R}_{+}$and $0<\lambda_{1}<\lambda_{2}$.

Proof. The first part of the statement follows from Theorem 2. Set

$$
\begin{array}{lll}
\underline{\varphi}_{+}(t ; \lambda)=K_{1}^{-1}\left(\lambda k_{1}(t)\right), & \bar{\varphi}_{+}(t ; \lambda)=K_{2}^{-1}\left(\lambda k_{2}(t)\right), \\
\underline{\varphi}_{-}(t ; \lambda)=V_{1}^{-1}\left(\lambda l_{1}(t)\right), & \bar{\varphi}_{-}(t ; \lambda)=V_{2}^{-1}\left(\lambda l_{2}(t)\right)
\end{array}
$$

for $t \in \mathbb{R}_{+}, \lambda>0$. Since $\left(18_{\lambda}\right)$ can be rewritten in the form

$$
\left(\frac{q(t)}{\lambda} k(u) u^{\prime}\right)=F(t, u) u^{\prime}, \quad \lambda>0,
$$

we have (see Lemma 3)

$$
\begin{aligned}
\left(21_{+}\right) u\left(t_{2}\right)-u\left(t_{1}\right) \geq & \lambda f_{1}\left(t_{2}\right) H_{1}\left(\underline{\varphi}_{+}\left(t_{1} ; \lambda\right)\right) \int_{t_{1}}^{t_{2}} \frac{d t}{q(t)} \\
& \times\left[\max \left\{k(x): \underline{\varphi}_{+}\left(t_{1} ; \lambda\right) \leq x \leq \bar{\varphi}_{+}\left(t_{2} ; \lambda\right)\right\}\right]^{-1}
\end{aligned}
$$


for any solution $u \in \mathcal{A}_{+}$of $\left(18_{\lambda}\right)$ and $0<t_{1}<t_{2}$, and

$$
\begin{aligned}
u\left(t_{1}\right)-u\left(t_{2}\right) \geq & -\lambda f_{2}\left(t_{2}\right) T_{2}\left(\bar{\varphi}_{-}\left(t_{1} ; \lambda\right)\right) \int_{t_{1}}^{t_{2}} \frac{d t}{q(t)} \\
& \times\left[\max \left\{-k(x): \underline{\varphi}_{-}\left(t_{2} ; \lambda\right) \leq x \leq \bar{\varphi}_{-}\left(t_{1} ; \lambda\right)\right\}\right]^{-1}
\end{aligned}
$$

for any solution $u \in \mathcal{A}_{-}$of $\left(18_{\lambda}\right)$ and $0<t_{1}<t_{2}$.

Set $\mathcal{K}_{\lambda, \varepsilon}=\left\{u \in \mathcal{A}_{\varepsilon}: \varphi_{\varepsilon}(t ; \lambda) \leq u(t) \leq \bar{\varphi}_{\varepsilon}(t ; \lambda)\right.$ for $t \in \mathbb{R}_{+}, u$ satisfies $\left.\left(21_{\varepsilon}\right)\right\}$ and define $\mathrm{T}_{\lambda, \varepsilon}: \mathcal{K}_{\lambda, \varepsilon} \rightarrow C^{0}\left(\mathbb{R}_{+}\right)$by

$$
\left(\mathrm{T}_{\lambda, \varepsilon} u\right)(t)=W_{\varepsilon}^{-1}\left(\lambda \int_{0}^{t} \frac{1}{q(s)} \int_{0}^{u(s)} F\left(u^{-1}(x), x\right) d x d s\right),
$$

where $\varepsilon \in\{+,-\}, \lambda>0$. Then (cf. Lemma 4) $\mathrm{T}_{\lambda, \varepsilon}: \mathcal{K}_{\lambda, \varepsilon} \rightarrow \mathcal{K}_{\lambda, \varepsilon}$. Next set

$$
\begin{array}{ll}
u_{\lambda, \varepsilon}^{[0]}(t)=\underline{\varphi}_{\varepsilon}(t ; \lambda), & u_{\lambda, \varepsilon}^{[n+1]}(t)=\left(\mathrm{T}_{\lambda, \varepsilon} u_{\lambda, \varepsilon}^{[n]}\right)(t), \\
v_{\lambda, \varepsilon}^{[0]}(t)=\bar{\varphi}_{\varepsilon}(t ; \lambda), & v_{\lambda, \varepsilon}^{[n+1]}(t)=\left(\mathrm{T}_{\lambda, \varepsilon} v_{\lambda, \varepsilon}^{[n]}\right)(t)
\end{array}
$$

for $t \in \mathbb{R}_{+}, n \in \mathbb{N}, \lambda>0$ and $\varepsilon \in\{+,-\}$. Then the limits

$$
\lim _{n \rightarrow \infty} u_{\lambda, \varepsilon}^{[n]}(t)=: u_{\varepsilon}(t ; \lambda), \quad \lim _{n \rightarrow \infty} v_{\lambda, \varepsilon}^{[n]}(t ; \lambda)=: v_{\varepsilon}(t ; \lambda)
$$

exist for $t \in \mathbb{R}, \lambda>0, \varepsilon \in\{+,-\}$.

Let $0<t_{1}<t_{2}$ and let for example $\varepsilon=+$ (for the case $\varepsilon=-$, the proof is similar). Then

$$
\underline{\varphi}_{+}\left(t ; \lambda_{1}\right)<\underline{\varphi}_{+}\left(t ; \lambda_{2}\right), \quad \bar{\varphi}_{+}\left(t ; \lambda_{1}\right)<\bar{\varphi}_{+}\left(t ; \lambda_{2}\right)
$$

and for each $\alpha, \beta \in \mathcal{A}_{+}$with $\alpha(t)<\beta(t)$ on $(0, \infty)$ we have

$$
\begin{aligned}
\left(\mathrm{T}_{\lambda_{1},+} \alpha\right)(t) & =W_{+}^{-1}\left(\lambda_{1} \int_{0}^{t} \frac{1}{q(s)} \int_{0}^{\alpha(s)} F\left(\alpha^{-1}(x), x\right) d x d s\right) \\
& <W_{+}^{-1}\left(\lambda_{2} \int_{0}^{t} \frac{1}{q(s)} \int_{0}^{\beta(s)} F\left(\beta^{-1}(x), x\right) d x d s\right)=\left(\mathrm{T}_{\lambda_{2},+} \beta\right)(t),
\end{aligned}
$$

and therefore

$$
u_{\lambda_{1},+}^{[n]}(t)<u_{\lambda_{2},+}^{[n]}(t), \quad v_{\lambda_{1},+}^{[n]}(t)<v_{\lambda_{2},+}^{[n]}(t) \quad \text { for } t \in(0, \infty), n \in \mathbb{N} .
$$

Hence

$$
u_{+}\left(t ; \lambda_{1}\right) \leq u_{+}\left(t ; \lambda_{2}\right), \quad v_{+}\left(t ; \lambda_{1}\right) \leq v_{+}\left(t ; \lambda_{2}\right) \quad \text { for } t \in \mathbb{R}_{+} .
$$

If $r\left(t_{0} ; \lambda_{1}\right)=r\left(t_{0} ; \lambda_{2}\right)$ for a $t_{0}>0$, where $r$ is either $u_{+}$or $v_{+}$, then $\left(r_{j}(t)=\right.$ $r\left(t ; \lambda_{j}\right)$ on $\left.\mathbb{R}_{+}, j=1,2\right)$ 


$$
\begin{aligned}
r_{1}\left(t_{0}\right) & =W_{+}\left(\lambda_{1} \int_{0}^{t_{0}} \frac{1}{q(s)} \int_{0}^{r_{1}(s)} F\left(r_{1}^{-1}(x), x\right) d x d s\right) \\
& <W_{+}\left(\lambda_{2} \int_{0}^{t_{0}} \frac{1}{q(s)} \int_{0}^{r_{2}(s)} F\left(r_{2}^{-1}(x), x\right) d x d s\right)=r_{2}\left(t_{0}\right),
\end{aligned}
$$

which is a contradiction.

TheOREM 7. Let $\int_{0}^{\infty} d s / q(s)<\infty$ and let assumptions $\left(\mathrm{H}_{1}\right)-\left(\mathrm{H}_{7}\right)$ be satisfied. Then for each $a \in \mathbb{R}, a \neq 0$, there exists a unique $\lambda_{0}>0$ such that equation $\left(18_{\lambda}\right)$ for $\lambda=\lambda_{0}$ has a (necessarily unique) solution $u\left(t ; \lambda_{0}\right)$ with

$$
\lim _{t \rightarrow \infty} u\left(t ; \lambda_{0}\right)=a .
$$

Proof. By Theorem 5 equation $\left(18_{\lambda}\right)$ has a unique solution $u_{+}(t ; \lambda) \in$ $\mathcal{A}_{+}$and a unique solution $u_{-}(t ; \lambda) \in \mathcal{A}_{-}$for each $\lambda>0$ and moreover the finite $\operatorname{limits} \lim _{t \rightarrow \infty} u_{+}(t ; \lambda)(>0)$ and $\lim _{t \rightarrow \infty} u_{-}(t ; \lambda)(<0)$ exist by Theorem 3. Define

$$
g_{+}(\lambda)=\lim _{t \rightarrow \infty} u_{+}(t ; \lambda), \quad g_{-}(\lambda)=\lim _{t \rightarrow \infty} u_{-}(t ; \lambda) \quad \text { for } \lambda>0 .
$$

Then $g_{+}:(0, \infty) \rightarrow(0, \infty)$ and $g_{-}:(0, \infty) \rightarrow(-\infty, 0)$. In view of Theorem $6, g_{+}$is increasing on $(0, \infty)$ and $g_{-}$is decreasing on $(0, \infty)$. If for example $g_{+}\left(\lambda_{1}\right)=g_{+}\left(\lambda_{2}\right)$ for some $0<\lambda_{1}<\lambda_{2}$, then setting $r_{1}(t)=u_{+}\left(t ; \lambda_{1}\right)$, $r_{2}(t)=u_{+}\left(t ; \lambda_{2}\right)$ for $t \in \mathbb{R}_{+}$we have $r_{1}(t)<r_{2}(t)$ on $(0, \infty), r_{1}^{-1}(t)>r_{2}^{-1}(t)$, $F\left(r_{1}^{-1}(t), t\right)<F\left(r_{2}^{-1}(t), t\right)$ for $t \in\left(0, g_{+}\left(\lambda_{1}\right)\right)$, hence

$$
\begin{aligned}
g_{+}\left(\lambda_{1}\right) & =\int_{0}^{\infty} \frac{1}{q(s)} \int_{0}^{r_{1}(s)} F\left(r_{1}^{-1}(x), x\right) d x d s \\
& <\int_{0}^{\infty} \frac{1}{q(s)} \int_{0}^{r_{2}(s)} F\left(r_{2}^{-1}(x), x\right) d x d s=g_{+}\left(\lambda_{2}\right),
\end{aligned}
$$

a contradiction. Consequently, $g_{+}$is strictly increasing, and $g_{-}$is strictly decreasing. To prove our theorem it is enough to show that $g_{+}$and $g_{-}$ map $(0, \infty)$ onto $(0, \infty)$ and $(-\infty, 0)$, respectively. We prove for example that $g_{+}$maps $(0, \infty)$ onto itself. First, we see from $\underline{\varphi}_{+}(t ; \lambda) \leq u_{+}(t ; \lambda) \leq$ $\bar{\varphi}_{+}(t ; \lambda)$, where $\underline{\varphi}_{+}, \bar{\varphi}_{+}$are defined by $(20)$, that $\lim _{\lambda \rightarrow 0_{+}} g_{+}(\lambda)=0$ and $\lim _{\lambda \rightarrow \infty} g_{+}(\lambda)=\infty$. Secondly, assume, on the contrary, $\lim _{\lambda \rightarrow \lambda_{0-}} g_{+}(\lambda)<$ $\lim _{\lambda \rightarrow \lambda_{0+}} g_{+}(\lambda)$ for a $\lambda_{0}>0$. Setting $v_{1}(t)=\lim _{\lambda \rightarrow \lambda_{0-}} u_{+}(t ; \lambda), v_{2}(t)=$ $\lim _{\lambda \rightarrow \lambda_{0+}} u_{+}(t ; \lambda)$ for $t \geq 0$, we get $v_{1} \neq v_{2}$. Using the Lebesgue dominated convergence theorem as $\lambda \rightarrow \lambda_{0-}$ and $\lambda \rightarrow \lambda_{0+}$ in the equality $\left(r_{\lambda}(t)=\right.$ $u_{+}(t ; \lambda)$ for $\left.(t ; \lambda) \in \mathbb{R}_{+} \times(0, \infty)\right)$ 


$$
r_{\lambda}(t)=W_{+}\left(\lambda \int_{0}^{t} \frac{1}{q(s)} \int_{0}^{r_{\lambda}(s)} F\left(r_{\lambda}^{-1}(x), x\right) d x d s\right)
$$

we see that

$$
v_{j}(t)=W_{+}\left(\lambda_{0} \int_{0}^{t} \frac{1}{q(s)} \int_{0}^{v_{j}(s)} F\left(v_{j}^{-1}(x), x\right) d x d s\right) \quad \text { for } t \geq 0, j=1,2 .
$$

Therefore $v_{1}, v_{2}$ are solutions of $\left(18_{\lambda}\right)$ for $\lambda=\lambda_{0}$, and consequently $v_{1}=v_{2}$, a contradiction.

\section{References}

[1] F. A. Atkinson and L. A. Peletier, Similarity profiles of flows through porous media, Arch. Rational Mech. Anal. 42 (1971), 369-379.

[2] - - - Similarity solutions of the nonlinear diffusion equation, ibid. 54 (1974), 373392.

[3] J. Bear, D. Zaslavsky and S. Irmay, Physical Principles of Water Percolation and Seepage, UNESCO, 1968.

[4] J. Goncerzewicz, H. Marcinkowska, W. Okrasiński and K. Tabisz, On the percolation of water from a cylindrical reservoir into the surrounding soil, Zastos. Mat. 16 (1978), 249-261.

[5] W. Okrasiński, Integral equations methods in the theory of the water percolation, in: Mathematical Methods in Fluid Mechanics, Proc. Conf. Oberwolfach, 1981, Band 24, P. Lang, Frankfurt/M, 1982, 167-176.

[6] -, On a nonlinear ordinary differential equation. Ann. Polon. Math. 49 (1989), $237-245$.

[7] S. Staněk, Nonnegative solutions of a class of second order nonlinear differential equations, ibid. 57 (1992), 71-82.

DEPARTMENT OF MATHEMATICAL ANALYSIS

FACULTY OF SCIENCE, PALACKÝ UNIVERSITY

TOMKOVA 38

77200 OLOMOUC, CZECHOSLOVAKIA 